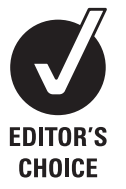

${ }^{1}$ Department of Health Policy and Management, Harvard School of Public Health, Boston, MA, USA ${ }^{2}$ Division of General Medicine, Brigham and Women's Hospital, Boston, MA, USA ${ }^{3}$ The VA Boston Healthcare System, Boston, MA, USA ${ }^{4}$ WHO World Alliance for Patient Safety, World Health Organization, Geneva, Switzerland

\section{Correspondence to}

Dr Ashish K Jha, Harvard School of Public Health, Boston,

Massachusetts, USA ajha@hsph.harvard.edu

Accepted 26 November 2008

\title{
Patient safety research: an overview of the global evidence
}

\author{
A K Jha, ${ }^{1,2,3}$ N Prasopa-Plaizier, ${ }^{4}$ I Larizgoitia, ${ }^{4}$ D W Bates, ${ }^{1,2}$ On Behalf of the Research \\ Priority Setting Working Group of the WHO World Alliance for Patient Safety
}

\section{ABSTRACT}

Background Unsafe medical care may cause substantial morbidity and mortality globally, despite imprecise estimates of the magnitude of the problem. To better understand the extent and nature of the problem of unsafe care, the WHO World Alliance for Patient Safety commissioned an overview of the world's literature on patient safety research.

Methods Major patient safety topics were identified through a consultative and investigative process and were categorised into the framework of structure, process and outcomes of unsafe care. Lead experts examined current evidence and identified major knowledge gaps relating to topics in developing, transitional and developed nations. The report was reviewed by internal and external experts and underwent improvements based on the feedback.

Findings Twenty-three major patient safety topics were examined. Much of the evidence of the outcomes of unsafe care is from developed nations, where prevalence studies demonstrate that between $3 \%$ and $16 \%$ of hospitalised patients suffer harm from medical care. Data from transitional and developing countries also suggest substantial harm from medical care. However,

considerable gaps in knowledge about the structural and process factors that underlie unsafe care globally make solutions difficult to identify, especially in resource-poor settings.

Interpretation Harm from medical care appears to pose a substantial burden to the world's population. However, much of the evidence base comes from developed nations. Understanding the scope of and solutions for unsafe care for the rest of the world is a critical component of delivering safe, effective care to all of the world's citizens.

Despite the longstanding principle to "do no harm", unsafe medical care appears to cause significant morbidity and mortality throughout the world. Although precise estimates are unavailable, prevalence studies from developed nations suggest that a substantial number of hospitalised patients are injured as a direct result of medical care. ${ }^{1-4}$ The evidence for harm in the ambulatory setting is much less robust but is likely to be sizeable. ${ }^{5} 6$ Generalising from these figures, tens of millions of people suffer injuries and millions likely die due to unsafe medical care. Injuries can occur in association with many medical interventions, from counterfeit or substandard drugs (due to regulatory and oversight failures) to healthcare-associated infections (due to unhygienic practices). Many of these injuries are preventable and, therefore, particularly troubling.
To better understand the causes and impact of the delivery of unsafe medical care from a global perspective, the World Health Organisation (WHO) Patient Safety team convened an ad hoc expert working group to establish priorities for research on patient safety. To help set priorities, the group commissioned a report on the current evidence available. This assessment was done by identifying topics in patient safety, examining related clinical and organisational issues and distinguishing gaps in current knowledge and directions for future research. This paper highlights the key points of the report. The full report, produced by the working group with the support of leading experts, is far more comprehensive ${ }^{7}$ and available on the WHO World Alliance for Patient Safety website (http:// www.who.int/patientsafety/research/en/).

\section{METHODS}

The group began by identifying the types and causes of adverse events that are particularly harmful to patients. Major patient safety issues were identified using a multi-faceted, iterative approach: we first began with a literature search. We identified the major causes of harm and their underlying causes. We then consulted with experts on the committee as well as with external sources, such as the National Patient Safety Foundation, reviews by the Agency for Healthcare Research and Quality $^{8}$ (which is the most comprehensive review of its kind) and epidemiologic studies from several nations including the US, ${ }^{9}$ Canada $^{1}$ and Australia. ${ }^{10}$ We went back to the experts on the committee with the preliminary list of harms and their underlying causes for further feedback. After finalising the list, we shared it with external experts to get any final feedback. With the list of harms and their underlying causes formalised (table 1), we then sought out experts in each individual topic area to write a section of the report on their topic of expertise.

While there is some debate about the relationship between quality and safety, the Institute of Medicine in the US suggests that safety is one critical component of the delivery of high-quality care. ${ }^{11}$ Therefore, the committee chose to categorise the patient safety topics identified into structure, process and outcomes. ${ }^{12}$ The Agency for Healthcare Research and Quality (AHRQ) defines "structure" as the resources and organisational arrangements in place to deliver care, "process" as the activities of providers for delivering care and "outcomes" as the consequences of clinical activities by providers. ${ }^{13}$

Each identified topic was then reviewed in detail by lead experts who were asked to describe the basic epidemiology of the topic (eg, frequency, 
Table 1 Major patient safety topics

\begin{tabular}{|c|c|c|}
\hline Topic \# & Quality domain & Patient safety topic \\
\hline 1 & Structure & Organisational determinants and latent failures \\
\hline 2 & Structure & $\begin{array}{l}\text { Structural accountability: the use of accreditation } \\
\text { and regulation to advance patient safety }\end{array}$ \\
\hline 3 & Structure & Safety culture \\
\hline 4 & Structure & $\begin{array}{l}\text { Inadequate training and education, manpower } \\
\text { issues }\end{array}$ \\
\hline 5 & Structure & Stress and fatigue \\
\hline 6 & Structure & Production pressures \\
\hline 7 & Structure & $\begin{array}{l}\text { Lack of appropriate knowledge and availability of } \\
\text { knowledge, transfer of knowledge }\end{array}$ \\
\hline 8 & Structure & $\begin{array}{l}\text { Devices, procedures without human factors } \\
\text { engineering }\end{array}$ \\
\hline 9 & Process & Errors in process of care through misdiagnosis \\
\hline 10 & Process & $\begin{array}{l}\text { Errors in the process of care through poor test } \\
\text { follow-up }\end{array}$ \\
\hline 11 & Process & $\begin{array}{l}\text { Errors in the structure and process of care: } \\
\text { counterfeit and substandard drugs }\end{array}$ \\
\hline 12 & Process & Measures of patient safety \\
\hline 13 & Process & Errors in process: unsafe injection practices \\
\hline 14 & Outcomes & $\begin{array}{l}\text { Adverse events and injuries due to medical } \\
\text { devices }\end{array}$ \\
\hline 15 & Outcomes & Adverse events due to medications \\
\hline 16 & Outcomes & $\begin{array}{l}\text { Adverse events: injury to patients due to surgical } \\
\text { errors }\end{array}$ \\
\hline 17 & Outcomes & $\begin{array}{l}\text { Adverse events due to healthcare associated } \\
\text { infections }\end{array}$ \\
\hline 18 & Outcomes & Adverse events due to unsafe blood products \\
\hline 19 & Outcomes & $\begin{array}{l}\text { Patient safety among pregnant women and } \\
\text { newborns }\end{array}$ \\
\hline 20 & Outcomes & Patient safety concerns among older adults \\
\hline 21 & Outcomes & Adverse events due to falls in the hospital \\
\hline 22 & Outcomes & Injury due to pressure sores and decubitus ulcers \\
\hline 23 & Outcomes & $\begin{array}{l}\text { How to bring the patients' voices into the patient } \\
\text { safety agenda }\end{array}$ \\
\hline
\end{tabular}

impact on care and preventability). These experts also identified major gaps in knowledge that need to be addressed through future research. Because healthcare systems, their focus and their impacts likely vary substantially based on the level of a nation's development, we asked experts to specify what is known about the individual topic in developing, transitional and developed nations. Nations were classified based on their category of national income (World Bank 2006 classification $^{14}$ ), and nations listed as "high income" were considered developed, "medium income" as transitional and "low income" as developing. Each section was reviewed by at least three other members of the committee for completeness and balance. Finally, the entire report underwent external review by seven international experts (names and affiliations listed in the Acknowledgement section).

In this manuscript, we synthesise the key points from the individual sections and present the major findings.

\section{RESULTS}

\section{Overview}

We identified 23 major patient safety topics for detailed examination (see table 1). Of these, eight were classified as structural, five as process and 10 as outcomes. While it was generally easier to separate the outcomes topics from others, the strong link between structural factors that underlie unsafe care and the unsafe processes they create necessarily lead to categorisation of some topics under one heading that could have arguably belonged in the other. The groupings were not meant to be definitive but created a practical framework for approaching the safety topics. Further, the topics covered activities and areas that could impact the health system at many different levels. For example, organisational accidents and failures obviously occur at the provider level and impact individual organisations such as hospitals or doctor practices. Counterfeit and substandard drugs, on the other hand, often represent failure to regulate and safeguard medications at the national level, impacting potentially all providers and patients in the country.

We found that unsafe patient care is common, that it is associated with significant morbidity and mortality throughout the world, and that much of the harm is likely amenable to intervention. Studies from the US suggest that approximately $3 \%$ to $4 \%$ of hospitalised patients suffer a serious adverse event, ${ }^{2}{ }^{3}$ while studies from other developed nations, using less restrictive definitions, found that between $8 \%$ and $16 \%$ of hospitalised patients suffer an adverse event. ${ }^{14}$ A substantial proportion (between $30 \%$ and $50 \%$ ) of adverse events are preventable, and most cause substantial harm, likely contributing to the deaths of tens of thousands of people in each of these nations. Although there have been few systematic prevalence studies in developing or transitional nations, existing evidence from these countries suggests that unsafe medical care is likely to be as-if not more-common ${ }^{15}{ }^{16}$ than in developed nations. For example, one study of Mexican hospitals suggested that nearly one in four hospitalised patients developed a nosocomial infection. ${ }^{15}$

The data on structural and process factors that affect patient safety come almost exclusively from a small number of developed nations. This makes understanding the underlying causes of unsafe care or recommending solutions to improve safety in developing and transitional nations extremely difficult. Even in developed nations, there are still substantial gaps in knowledge about the structural and process factors that underlie unsafe care. We outline the key findings, beginning with these factors, and then the outcomes of such care.

\section{Structural factors contributing to unsafe care}

A major contributor to unsafe care is the breakdown of complex systems, which some have called "organisational accidents". These breakdowns arise from combinations of factors originating at different levels of the system and can involve latent failures or poor oversight. ${ }^{17}$ A key structural issue that impacts safety is the inadequate number of qualified healthcare providers worldwide. Globally, 57 countries have an estimated deficit of 2.4 million doctors, nurses and midwives ${ }^{18}$ and thus face substantial challenges in meeting health-related Millennium Development Goals for improving the quality and safety of their healthcare systems. A closely related issue to inadequate staffing is production pressures, which refer to situations in which the optimal patient care capacity of a healthcare system or an individual healthcare provider has been exceeded. Providing for too many patients at one time distracts providers, forces greater reliability on memory to perform important actions and hinders effective communication among healthcare personnel. Each of these likely creates an environment for unsafe processes. For example, one analysis in 2005 identified communication problems as the single biggest cause of nearly $70 \%$ of sentinel events in the hospital setting in the US. ${ }^{19}$ The roles of organisational structure, capacity and communication breakdowns in producing unsafe care in developing and transitional countries have not been adequately studied.

A related threat hindering the delivery of safe care is provider fatigue. Doctors-in-training who work traditional 24-h shifts make $36 \%$ more serious medical errors in the care of their patients than comparable doctors not doing extended shift 
work. Fatigued doctors make up to five times as many serious diagnostic errors, report making four times as many fatiguerelated errors that lead to a patient's death and suffer many more occupational injuries themselves. ${ }^{20-23}$ Although data on provider fatigue come primarily from developed nations, providers in developing and transitional nations are likely to be at least as susceptible to these threats.

We found that other key structural issues that may affect safety include the organisation's patient safety culture, which refers to shared attitudes, values and norms related to safety. A positive culture may result in improvements in safety practices through better communication, teamwork and knowledge sharing, although the evidence base underlying this assumption is weak. Further, there is scarce knowledge about how organisational factors combine with provider factors to affect patient safety culture. Another important topic includes the role that accreditation and regulations play in improving accountability and systems of care. While both are felt to likely impact safety, their actual level of influence on patient safety has not been empirically assessed and their roles are especially uncertain in developing and transitional countries.

Finally, human factors engineering (HFE) represents an important structural issue to understand the hazards of medical care and ways to minimise those risks. HFE techniques and heuristics can also assist in investigating adverse events ${ }^{24}$ when they do occur. Their primary value has been in improving troublesome design issues involving architecture, devices and clinical procedures $^{25}$ (eg, anaesthesiology, ${ }^{26}$ surgery $^{27}$ and nursing ${ }^{28}$ ). By understanding how individuals actually interface with technology, for example, HFE can reduce adverse event rates by maximising the human ability to use technology effectively.

\section{Processes that underlie unsafe care}

Misdiagnosis is understudied but represents a major type of error in healthcare with widely ranging rates of delayed and erroneous diagnosis. Even in the most highly developed countries with sophisticated technology, at least $10 \%$ to $15 \%$ of diagnoses are incorrect. ${ }^{29}$ The numbers from developing and transitional countries are surely higher and likely add substantial financial costs and create significant morbidity and mortality. For example, one review found high rates of overdiagnosis of malaria in developing nations with consequent underdiagnosis of pneumonia and other related disorders, leading to undertreatment and likely high rates of morbidity from the underlying condition. ${ }^{30}$ Another important failure of process is the lack of adequate follow-up of important tests. Data from developed countries suggest that only about half of critically important laboratory results indicating potentially life-threatening conditions were followed up by appropriate treatment in a timely manner. ${ }^{31}$ The rates of test follow-up in developing nations are also suboptimal and variable and cause serious lapses in patient care. ${ }^{32}$

Counterfeit and substandard drugs, defined as those that are mislabelled, missing active ingredients or include wrong active ingredients, pose a major risk to patient safety. It was classified by the group under unsafe processes (due to poor regulatory oversight of medication safety) but could be considered a structural failure of the healthcare system. Repeated use of counterfeit or substandard medicines can result in therapeutic failure, drug resistance or even death. ${ }^{33} 34$ Counterfeit drugs account for more than $10 \%$ of the global medicine market and up to $30 \%$ of medicines consumed in developing countries. ${ }^{33} 35$ It is likely that hundreds of thousands of people, if not more, die each year due to consumption of substandard medications, ${ }^{33}$ but the precise burden of the problem is unknown.
Unsafe injection practices also cause substantial morbidity and mortality in large parts of the world. In 2000, WHO estimated that some 16 billion injections were administered each year in transitional and developing nations and up to $40 \%$ of injections were given with syringes and needles reused without sterilisation; in some countries, this proportion was as high as $70 \%{ }^{36}$ These obviously contribute to high rates of infections with hepatitis viruses, human immunodeficiency virus (HIV) and other transmittable disease.

\section{Outcomes of unsafe care}

Adverse events can occur as a result of nearly any interaction with the healthcare system. Estimates from developed nations suggest that between $7.5 \%$ and $10.4 \%$ of hospitalised patients experience injuries due to medications alone. ${ }^{13738}$ These adverse drug events (ADEs) cost tens of billions of dollars to healthcare systems around the world and have been estimated to contribute to 140000 deaths each year in the US alone. ${ }^{39} 40$ Best estimates suggest that $28 \%$ to $56 \%$ of $\mathrm{ADEs}$ are preventable. ${ }^{41}$ The rates of $\mathrm{ADEs}$ from developing and transitional nations are largely unknown. Although the official rates of medication use are much lower in developing and transitional countries, the actual use of medications in these nations is hard to quantify. ${ }^{42}$ Therefore, whether $\mathrm{ADEs}$ are less common in these nations is unknown but they likely represent a major source of patient harm and economic costs.

The best evidence suggests that medical devices can also cause substantial harm. Errors that underlie device-related injuries are often categorised into three groups: manufacturer-related errors, user errors and use or design errors. ${ }^{43-50}$ In the US, more than 1 million adverse medical device events occur annually, at a rate of 6.3 events per 1000 patient days. ${ }^{51}$ Studies by WHO suggest that adverse medical device events might be particularly problematic in developing countries, where medical equipment is often improperly maintained or replaced, placing patients at great risk. ${ }^{52}$ One study from a transitional nation found that the rates of infection from medical devices alone were 34.2 per 1000 patient days in the hospital. ${ }^{53}$

Surgery and anaesthesia also present substantial safety risks. In the US, estimates suggest that surgical adverse events account for $48 \%$ of all adverse events and are preventable $54 \%$ to $74 \%$ of the time. ${ }^{254}$ The few available studies from the developing world have found surgical adverse event rates to be as much as fivefold to 10-fold higher. ${ }^{55-60}$ Improving the use of evidence-based practices could potentially reduce these rates dramatically. ${ }^{61}$

Nosocomial infections are reported to occur in approximately $5 \%$ to $10 \%$ of hospitalised patients in developed nations ${ }^{15}$ and between $25 \%$ and $40 \%$ in developing nations. ${ }^{15} 16$ One in four patients in intensive care may acquire an infection during a stay in hospital and one estimate suggests that these rates are twice as high in developing countries. ${ }^{16}$ Common types of nosocomial infections include nosocomial pneumonia, catheter-related infections and surgical infections. These events are not only common but also highly preventable. They represent a major source of morbidity and mortality, as well as substantial associated financial costs to health systems.

Another important source of infections from medical care is the use of unsafe blood products. Recent estimates indicate that $5 \%$ to $15 \%$ of HIV infections in developing countries result from unsafe blood transfusion. ${ }^{62}{ }^{63}$ Unsafe blood poses a high risk for transmission of other blood-borne infections including hepatitis B, hepatitis C, syphilis, malaria, Chagas disease and West Nile fever. Studies demonstrate that nearly three in five countries lack an established quality system to screen collected blood for HIV 
and that $88.5 \%$ of blood units in sub-Saharan Africa are not screened for HIV in a reliable manner. ${ }^{64} 65$ It is not known what fraction of blood products in the developing world is tainted. Safe blood products are a particular concern for women of childbearing age, for whom severe haemorrhaging is a leading cause of maternal mortality.

\section{Outcomes of unsafe care in vulnerable populations}

With an estimated 7.6 million infant deaths during the perinatal period each year and approximately 600000 deaths in women due to pregnancy or childbirth ( $99 \%$ of which occur in developing countries), maternal and child health remains a major concern worldwide. ${ }^{66-68}$ Although many such deaths result from lack of access to care, many are also due to unsafe care. No well-designed studies address what fraction of the morbidity and mortality of women and newborns is attributable to a lack of access to care or to the receipt of unsafe, poor-quality care. Given the importance of understanding the causes of high maternal mortality in developing nations, deciphering the role that unsafe medical care plays is critically important.

The older people are particularly vulnerable to adverse events. Falls, for example, represent the most common patient safety injury for the older patients in hospitals in developed nations. ${ }^{69}$ Hip fractures remain common and only $14 \%$ to $21 \%$ of patients recover the ability to perform daily activities. ${ }^{70}$ Decubitus ulcers are also widespread in this group, risk factors of which include immobility, friction, incontinence, cognitive impairment and poor nutritional status. ${ }^{71-73}$ In the US between 1990 and 2001, decubitus ulcers were reported to be the cause of death for 114380 persons (age-adjusted mortality rate, 3.79 per 100000 population). ${ }^{74}$ Additionally, rates of ADEs among older patients are much higher than in the general population. ${ }^{75} 76$

\section{DISCUSSION}

We examined the available research on patient safety and focused specifically on 23 topics and have summarised the major consequences of unsafe care and its underlying causes. Several key findings emerged. First, the available data suggest that harm from medical care is widespread and likely imposes a substantial burden on the world's population. Second, most evidence about safety comes from developed nations, although there is growing epidemiological evidence of poor clinical outcomes due to unsafe medical care in developing and transitional countries. Finally, the data on structural and process factors that contribute to unsafe medical care are almost exclusively from a small number of developed nations. Although some solutions are readily apparent, large gaps in knowledge need to be filled before more comprehensive solutions can be developed, particularly for transitional and developing countries.

The nature and extent of unsafe care are still poorly understood in developing and transitional nations. For 5 of 10 "outcomes" of unsafe care (ADEs, adverse medical device events, surgical errors, falls and decubitus ulcer), there were few data points from developing or transitional nations. Data suggest that the burden of harm from unsafe care is sizeable in developed nations and likely to be comparable if not greater in transitional and developing nations. However, the lack of more reliable information underscores the need for high-quality epidemiological studies from these nations. Even among areas with a known level of harm, such as tainted blood products and nosocomial infections, research is needed to better understand the burden they pose to the population and national health systems and the efficacy of existing prevention and harm minimisation strategies.
In developed nations too, information is still lacking in many areas. We know relatively little about harm outside the hospital or who among the chronically ill is at particular risk for developing decubitus ulcers. We still need to develop many more effective intervention strategies. For all nations, the role of organisational structures and processes is poorly understood. Although inadequate numbers of high-quality staff affect the safety of care, optimal staffing levels and the appropriate mix of skills is not known. How organisational and provider factors combine to affect patient safety needs greater understanding.

To ensure comparability of data across the world, standardised tools, measures and definitions are needed. The WHO World Alliance for Patient Safety has focused on this area. ${ }^{77}$ Beyond definitions, as practice patterns change, we will need robust mechanisms for ongoing data collection from a range of nations, especially developing and transitional ones. The potential benefits of improving safety are enormous. For example, studies suggest that establishing safe injection practices around the globe could prevent as many as 1.3 million early deaths each year and eliminate billions of dollars (US\$) annually in direct medical costs. $^{78}$ The primary implications for funders of healthcare are that we still need both new evidence about the extent of harm that occurs from unsafe medical care in developing countries, the impact that harm has on patients' distrust of those systems, and we need solutions that are able to be implemented locally and are cost-effective.

This study has important limitations. First, although we attempted to account for the most important topics in patient safety, due to constraints, we addressed only those that account for the most harm and some topics that are also important could not be addressed. Although this report could not be wholly comprehensive and was not meant to be a series of systematic reviews of each topic, we do believe we captured most of the major issues in safety and identified the main literature in these areas. Another important limitation was a lack of reliable data in many of the areas we covered, especially for developing countries. Although the limited available information suggests that the burden of harm from unsafe care is very large, it will be essential to obtain more reliable data. Further, whether the key lessons learnt from the developed nations are applicable to the developing world is largely unknown. Next, as has been mentioned previously, many of the topics could have been categorised into multiple areas (ie, structure or process) and those categorisations were not meant to be definitive. Finally, in the interest of brevity, we necessarily omitted many details that are available in the larger report.

In conclusion, patients seek care to reduce their suffering. Based on research from the past two decades, we know that while the healthcare system cures disease and alleviates pain, it can also cause largely preventable harm and suffering. This evidence should not be interpreted as an acceptable cost of providing healthcare. Our review suggests that harm occurs too often and that much of it is avoidable. Reducing harm will require targeted, well-designed and appropriately managed research to gain greater understanding of its causes and contributing factors, especially in transitional and developing countries. The next generation of research should therefore focus both on better definitions of the problem and on effective solutions that reduce harm in medical care.

Acknowledgements The contributors to the report: Linda Aiken, University of Pennsylvania; Benedetta Allegranzi, WHO World Alliance for Patient Safety; Roselie Bright, USA Food and Drug Administration; Eric Campbell, Massachusetts General Hospital; Richard Cooper, University of Pennsylvania; Neelam Dhingra-Kumar, World Health Organization; Björn Fahlgren, World Health Organization; Timothy Ferris, 
Massachusetts General Hospital and Harvard Medical School; Terry Field, Meyers Primary Care Institute and University of Massachusetts Medical School; John Gosbee, Red Forest Consulting and University of Michigan Health System; Daniel Grandt, Hospital of Saarbrücken; Jerry Gurwitz, Meyers Primary Care Institute and University of Massachusetts Medical School; Tom Isaac, Veterans Administration Boston Healthcare System; Allen Kachalia, Brigham and Women's Hospital; Selma Khamassi, World Health Organization; Barrett Kitch, Brigham and Women's Hospital and Harvard Medical School; Christopher Landrigan, Children's Hospital, Boston; Michael Matheny, Partners Healthcare System and Brigham and Women's Hospital; Saverio Maviglia, Partners Healthcare System; Mario Merialdi, World Health Organization; Harvey Murff, Veterans Administration Healthcare System and Vanderbilt Epidemiology Center; Eric Poon, Brigham and Women's Hospital; Jim Reason, University of Manchester; Gordon Schiff, Cook County Hospital; Ryan Sidorchuk, Winnipeg Regional Health Authority; Thomas Wuerz, New England Medical Centre

The report reviewers: Ross Baker, University of Toronto; N K Ganguly, Indian Council of Medical Research; Tawfik Khoja, Health Ministers Council for Gulf Cooperation Council States; Niek Klazinga, University of Amsterdam; John Ovretveit, Karolinska Institutet; Susan Sheridan, Consumers Advancing Patient Safety; Lucian Leape, Harvard School of Public Health; Hilary Coates, Health Information and Quality Authority of Ireland; Martin Fletcher, National Patient Safety Agency of England and Wales; and the World Health Organization officers: Meena Nathan Cherian, Gerald Dziekan, Jan Fordham, Helen Hughes, Mathews Mathai and Ramesh Shademani. The Research Priority Setting Working Group of the WHO World Alliance for Patient Safety include: Benedetta Allegranzi, World Health Organization; Peter Angood, Joint Commission on Accreditation of Health Care Organizations; Zulfiqar Bhutta, Aga Khan University; Peter Davis, University of Auckland; Daniel Grandt, Hospital of Saarbrücken; Maimunah Hamid, Institute for Health Systems Research of Thailand; Jorge Insua, Hospital Universitario Austral; Robinah Kaitiritimba, Uganda National Health Consumers Organization; Selma Khamassi, World Health Organization; Thandinkosi Madiba, University of Kwazulu-Natal; Takeshi Morimoto, Kyoto University; Douglas Noble, World Alliance for Patient Safety; Peter Norton, University of Calgary; Tikki Elka Pang, World Health Organization; Ryan Sidorchuk, Winnipeg Regional Health Authority; Anuwat Supachutikul, Institute of Hospital Quality Improvement and Accreditation of Thailand; Eric Thomas, University of Texas at Houston; and David Bates (Chair), Ashish Jha, Itziar Larizgoitia and Nittita PrasopaPlaizier, World Alliance for Patient Safety, World Health Organization as secretariat. We thank Ms Jordana Nunes Miranda and Ms Katthyana Aparicio for their assistance with this work.

The full report is available at http://www.who.int/patientsafety/research/en.

Funding The project was funded by the WHO World Alliance for Patient Safety, World Health Organization, Geneva, Switzerland.

Competing interests None.

\section{REFERENCES}

1. Baker GR, Norton PG, Flintoft V, et al. The Canadian Adverse Events Study: the incidence of adverse events among hospital patients in Canada. CMAJ 2004;170:1678-86.

2. Brennan TA, Leape LL, Laird NM, et al. Incidence of adverse events and negligence in hospitalized patients. Results of the Harvard Medical Practice Study I. N Engl J Med 1991;324:370-6.

3. Thomas EJ, Studdert DM, Burstin HR, et al. Incidence and types of adverse events and negligent care in Utah and Colorado. Med Care 2000;38:261-71.

4. Wilson RM, Runciman WB, Gibberd RW, et al. The quality in Australian health care study. Med J Aust 1995;163:458-71.

5. Gandhi TK, Weingart SN, Borus J, et al. Adverse drug events in ambulatory care. N Engl J Med 2003:348:1556-64.

6. Sequist TD, Bates DW, Cook EF, et al. Prediction of missed myocardial infarction among symptomatic outpatients without coronary heart disease. Am Heart $J 2005$ 149:74-81.

7. Jha A, ed. Summary of the evidence on patient safety: implications for research. Geneva: World Health Organization, 2008:xiil.

8. Warren DK, Cosgrove SE, Diekema DJ, et al. A multicenter intervention to prevent catheter-associated bloodstream infections. Infect Control Hosp Epidemiol 2006:27:662-9

9. Leape LL, Brennan TA, Laird N, et al. The nature of adverse events in hospitalized patients. Results of the Harvard Medical Practice Study II. N Engl J Med 1991;324:377-84.

10. Wilson RM, Runciman WB, Gibberd RW, et al. Quality in Australian health care study. Med J Aust 1996;164:754.

11. Corrigan JM, Donaldson MS, Kohn LT, eds. Crossing the quality chasm: a new health system for the 21st century. Washington (DC): National Academy Press: 2001:1-22.

12. Donabedian A. Evaluating the quality of medical care. Milbank Mem Fund 0 1966;44 (3 Suppl):166-206

13. Child Health Care Quality Toolbox: Measuring quality in children's health. Understanding quality measurement. Agency for Healthcare Research and Quality, 2004. http://www.ahrq.gov/chtoolbx/understn.htm (accessed 10 August 2006).

14. World Bank. Data and statistics: country classification. Washington, DC: World Bank, 2006. http://web.worldbank.org/WBSITE/EXTERNAL/DATASTATISTICS/0,
contentMDK:20420458 menuPK:64133156 pagePK:64133150 piPK: 64133175 theSitePK:239419,00.html (accessed 10 August 2006).

15. Global patient safety challenge launched. http://www.findarticles.com/p/articles/ mi mOFSL/is 1 83/ai n16100927/print (accessed 10 August 2006).

16. Moreno CA, Rosenthal VD, Olarte N, et al. Device-associated infection rate and mortality in intensive care units of 9 Colombian hospitals: findings of the International Nosocomial Infection Control Consortium. Infection Control and Hospital Epidemiology 2006;27:349-56

17. Reason J. Safety in the operating theatre - Part 2: human error and organisational failure. Qual Saf Health Care 2005;14:56-60.

18. WHO. Working together for health: the world health report 2006. Geneva, Switzerland WHO Press, 2006.

19. Sentinel Event Statistics. 2006. http://www.jointcommission.org/SentinelEvents/ Statistics (accessed 17 August 2006)

20. Landrigan CP, Rothschild JM, Cronin JW, et al. Effect of reducing interns' work hours on serious medical errors in intensive care units. N Engl J Med 2004; 351:1838-48.

21. Barger LK, Cade BE, Ayas NT, et al. Extended work shifts and the risk of motor vehicle crashes among interns. N Engl J Med 2005;352:125-34.

22. Lockley SW, Cronin JW, Evans EE, et al. Effect of reducing interns' weekly work hours on sleep and attentional failures. N Engl J Med 2004;351:1829-37.

23. Ayas NT, Barger LK, Cade BE, et al. Extended work duration and the risk of selfreported percutaneous injuries in interns. JAMA 2006:296:1055-62.

24. Zhang J, Johnson TR, Patel VL, et al. Using usability heuristics to evaluate patient safety of medical devices. J Biomed Inform 2003;36:23-30.

25. Graham MJ, Kubose TK, Jordan D, et al. Heuristic evaluation of infusion pumps: implications for patient safety in Intensive Care Units. Int J Med Inform 2004;73:771-9.

26. Gaba DM. Anaesthesiology as a model for patient safety in health care. BMJ 2000:320:785-8.

27. Carthey J, de Leval MR, Reason JT. The human factor in cardiac surgery: errors and near misses in a high technology medical domain. Ann Thorac Surg 2001;72:300-5.

28. Etchells E, Bailey C, Biason R, et al. Human factors in action: getting "pumped" at a nursing usability laboratory. Healthc 0 2006;9:69-74.

29. Graber ML, Franklin N, Gordon R. Diagnostic error in internal medicine. Arch Intern Med 2005:165:1493-9.

30. Amexo M, Tolhurst R, Barnish G, et al. Malaria misdiagnosis: effects on the poor and vulnerable. Lancet 2004;364:1896-8.

31. Tate KE, Gardner RM, Weaver LK. A computerized laboratory alerting system. MD Comput 1990; 7:296-301.

32. Murff H, Bates D. Notifying patients of abnormal results. In:Shojania K, Duncan B McDonald K, et al, eds. Making health care safer: a critical analysis of patient safety practices. Rockville (MD): Agency for Healthcare Research and Quality, 2001:i-vi.

33. Substandard and counterfeit medicines, Fact sheet 275. World Health Organization, 2003. http://www.who.int/mediacentre/factsheets/2003/fs275/en/. (accessed 10 August 2006).

34. Counterfeit medicines: some frequently asked questions. World Health Organization, 2005. Fact sheet. 2005. http://www.wpro.who.int/media_centre/ fact sheets/fs 20050506.htm (accessed 10 August 2006).

35. WHO. Counterfeit medicines: an update on estimates. Geneva, Switzerland: World Health Organization, 2006.

36. Hauri AM, Armstrong GL, Hutin YJ. The global burden of disease attributable to contaminated injections given in health care settings. Int J STD AIDS 2004;15:7-16.

37. Miller GC, Britth HC, Valenti L. Adverse drug events in general practice patients in Australia. Med J Aust 2006; 184:321-4.

38. Bates DW, Cullen DJ, Laird N, et al. Incidence of adverse drug events and potential adverse drug events. Implications for prevention. ADE Prevention Study Group. JAMA 1995:274:29-34.

39. Runciman WB, Roughead EE, Semple SJ, et al. Adverse drug events and medication errors in Australia. Int J Qual Health Care 2003:15 Suppl 1:i49-59.

40. Classen DC, Pestotnik SL, Evans RS, et al. Adverse drug events in hospitalized patients. Excess length of stay, extra costs, and attributable mortality. JAMA 1997;277:301-6.

41. Raschke RA, Gollihare B, Wunderlich TA, et al. A computer alert system to prevent injury from adverse drug events: development and evaluation in a community teaching hospital. JAMA 1998:280:1317-20.

42. IMSHealth. Global pharmaceutical sales by region. 2005. updated 2006 February 25 http://www.imshealth.com/ims/portal/front/articleC/0,00.html, 2777,6599 7747857977479643 (accessed 17 August 2006)

43. Amoore J, Ingram P. Learning from adverse incidents involving medical devices. Nurs Stand 2003;17:41-6.

44. Gosbee J. Introduction to the human factors engineering series. Jt Comm J Qual Saf 2004;30:215-9.

45. Render ML. Research and redesign are safer than warnings and rules. Crit Care Med 2004:32:1074-5.

46. Lin L, Vicente KJ, Doyle DJ. Patient safety, potential adverse drug events, and medical device design: a human factors engineering approach. J Biomed Inform 2001;34:274-84.

47. Welch DL. Human factors in the health care facility. Biomed Instrum Technol 1998;32:311-6

48. Mosenkis R. Human factors in design. In:van Gruting C, ed. Medical devices: international perspectives on health and safety. New York: Elsevier 1994:41-51.

49. Ward JR, Clarkson PJ. An analysis of medical device-related errors: prevalence and possible solutions. J Med Eng Technol 2004:28:2-21.

50. Bogner $\mathbf{M}$, ed. Human error in medicine Mahwah (NJ): Lawrence Erlbaum Assoc Inc 1994:1-12 
51. Bright RA, Brown SL. Medical device epidemiology. In:Brown SL, Bright RA, Tavris DR, eds. Medical device epidemiology and surveillance England (UK): John Wiley \& Sons Ltd 2007:27.

52. Pittet D, Donaldson L. Challenging the world: patient safety and health careassociated infection. Int J Qual Health Care 2006;18:4-8.

53. Inan D, Saba R, Yalcin AN, et al. Device-associated nosocomial infection rates in Turkish medical-surgical intensive care units. Infection Control and Hospital Epidemiology 2006:27;343-8.

54. Gawande AA, Thomas EJ, Zinner MJ, et al. The incidence and nature of surgical adverse events in Colorado and Utah in 1992. Surgery 1999;126:66-75.

55. Hernandez K, Ramos E, Seas C, et al. Incidence of and risk factors for surgical-site infections in a Peruvian hospital. Infect Control Hosp Epidemiol 2005;26:473-7.

56. Soleto L, Pirard M, Boelaert M, et al. Incidence of surgical-site infections and the validity of the National Nosocomial Infections Surveillance System risk index in a general surgical ward in Santa Cruz, Bolivia. Infect Control Hosp Epidemiol 2003;24:26-30.

57. Bratzler DW, Houck PM. Antimicrobial prophylaxis for surgery: an advisory statement from the National Surgical Infection Prevention Project. Am J Surg 2005:189:395-404.

58. Delgado-Rodriguez M, Sillero-Arenas M, Medina-Cuadros M, et al. Nosocomial infections in surgical patients: comparison of two measures of intrinsic patient risk. Infect Control Hosp Epidemiol 1997;18:19-23.

59. Coello R, Charlett $\mathrm{A}$, Wilson $\mathrm{J}$, et al. Adverse impact of surgical site infections in English hospitals. J Hosp Infect 2005;60:93-103.

60. Koigi-Kamau R, Kabare LW, Wanyoike-Gichuhi J. Incidence of wound infection after caesarean delivery in a district hospital in central Kenya. East Afr Med J 2005;82:357-61.

61. WHO. Second global patient safety challenge: safe surgery saves lives. 2008. http:// www.who.int/patientsafety/challenge/second gpsc/en/index.html laccessed 14 August 2006)

62. Lackritz EM. Prevention of HIV transmission by blood transfusion in the developing world: achievements and continuing challenges. AIDS 1998;12 Suppl A:S81-6.

63. Wake DJ, Cutting WA. Blood transfusion in developing countries: problems, priorities and practicalities. Trop Doct 1998;28:4-8.
64. Statement by Dr. Neelam Dhingra, Coordinator, Blood Transfusion Safety, World Health Organization: "Making Safe Blood Available in Africa". Committee on International Relations Subcommittee on Africa, Global Human Rights, and International Operations, US House of Representatives 2006.

65. WHO. Global database on blood safety, 2001-2002. Geneva (Switzerland): World Health Organization, 2004

66. Shah D, Shroff S, Sheth S. Reproductive and sexual health and safe motherhood in the developing world. Eur J Contracept Reprod Health Care 1999;4:217-28.

67. Lawn JE, Cousens S, Zupan J. 4 million neonatal deaths: when? Where? Why? Lancet 2005; 365:891-900.

68. WHO. Reproductive health strategy. Geneva (Switzerland): Reproductive Health and Research, 2004

69. Schwendimann R, Buhler $\mathrm{H}$, De Geest $\mathrm{S}$, et al. Falls and consequent injuries in hospitalized patients: effects of an interdisciplinary falls prevention program. BMC Health Serv Res. 2006;6:69. [electronic resource].

70. Zuckerman JD. Hip fracture. N Engl J Med. 1996;334:1519-25.

71. Berlowitz DR, Wilking SV. Risk factors for pressure sores. A comparison of crosssectional and cohort-derived data. J Am Geriatr Soc 1989;37:1043-50.

72. Brandeis GH, Ooi WL, Hossain M, et al. A longitudinal study of risk factors associated with the formation of pressure ulcers in nursing homes. J Am Geriatr Soc 1994; 42:388-93

73. Allman RM, Goode PS, Patrick MM, et al. Pressure ulcer risk factors among hospitalized patients with activity limitation. JAMA 1995:273:865-70.

74. Redelings MD, Lee NE, Sorvillo F. Pressure ulcers: more lethal than we thought? Adv Skin Wound Care 2005;18:367-72.

75. Gurwitz JH, Field TS, Harrold LR, et al. Incidence and preventability of adverse drug events among older persons in the ambulatory setting. JAMA 2003;289:1107-16.

76. Gurwitz JH, Field TS, Avorn J, et al. Incidence and preventability of adverse drug events in nursing homes. Am J Med 2000;109:87-94.

77. Global patient safety challenge launched. http://www.findarticles.com/p/articles/ mi mOFSL/is 1 83/ai n16100927/print laccessed 10 August 2006).

78. Miller MA, Pisani E. The cost of unsafe injections. Bull World Health Organ 1999:77:808-11. 